\title{
Precision Evaluation of the DC Decay Method for Accurate Characterization of Wound-Field Synchronous Machines and Comparison with the Sudden Short-Circuit
}

This paper was downloaded from TechRxiv (https://www.techrxiv.org).

\section{LICENSE}

CC BY 4.0

SUBMISSION DATE / POSTED DATE

22-10-2021 / 26-10-2021

\section{CITATION}

Maurer, Frederic; Nøland, Jonas Kristiansen (2021): Precision Evaluation of the DC Decay Method for Accurate Characterization of Wound-Field Synchronous Machines and Comparison with the Sudden ShortCircuit. TechRxiv. Preprint. https://doi.org/10.36227/techrxiv.16860517.v1

$\mathrm{DOI}$ 


\title{
Precision Evaluation of the DC Decay Method for Accurate Characterization of Wound-Field Synchronous Machines and Comparison with the Sudden Short-Circuit
}

\author{
Frederic Maurer, and Jonas Kristiansen Nøland, Member, IEEE
}

\begin{abstract}
The sudden short-circuit is considered the gold-standard parameter measurement method for wound-field synchronous machines (WFSMs) as it enables the recording of the characteristic quantities in near-to-real conditions. However, the test needs huge pieces of equipment, but even worse, it reduces the lifetime of the electrical components by up to 10 years due to the high winding overhang mechanical forces. The DC-Decay tests are low-power alternatives to obtain the characteristic quantities without damaging the machinery. To allow wider use of this method, there are a couple of challenges left that are tackled by this paper. The two main open challenges are, firstly, the number of measurements needed to reach a particular precision, and secondly, a comparison of the DC-Decay with the sudden short-circuit test to allow the validation against the gold standard. More detailed, this paper explores the main challenges associated with the practical use of the DC decay method, which is a non-conventional and detailed-level approach to characterize WFSMs. We provide replies and recommendations regarding the number of measurements, suggesting the minimum number of recorded tests needed to obtain the equivalent diagram with a given accuracy, which has been further validated with an experimental case study. Moreover, the potential enhancement and precision of the parameter identification algorithm are studied in detail. Finally, the equivalent parameters of the DC decay method are compared to the gold standard, which concludes on what the characterization means in terms of predicting accurate transient short-circuit currents for WFSMs.
\end{abstract}

Index Terms-DC decay methods, parameter identification, pole estimation, equivalent diagrams, synchronous machines, current measurement, transient analysis, three-phase short-circuit.

\section{NOMENCLATURE}

$T_{d 0}^{\prime}, T_{d 0}^{\prime \prime}, T_{q 0}^{\prime \prime}$

$T_{d}^{\prime}, T_{d}^{\prime \prime}, T_{q}^{\prime \prime}$

$\theta$

$T_{\sigma D}, T_{\sigma f}$

$\omega_{n}$

$x_{a d}, x_{a q}$

$x_{c}$

$x_{d}, x_{d}^{\prime}, x_{d}^{\prime \prime}$

$x_{q}, x_{q}^{\prime \prime}$

$i_{a}, i_{b}, i_{c}$

$i_{D}, i_{Q}$

$i_{d}, i_{q}$

$i_{f}$

$r_{D}, r_{Q}$

$r_{s}, r_{f}$

$s$

$s_{1}, s_{2}, s_{3}, s_{4}$

$u_{d}, u_{q}$

Trans. \& subtrans. open-circuit time const., $[s]$ Trans. \& subtrans. short-circuit time const., $[s]$ Angle between rotor \& stator phase $a,[\mathrm{rad}]$ Leakage time const. damper \& field, $[s]$ Rated pulsation, $2 \pi f_{n},[\mathrm{rad} / \mathrm{s}]$ Main d- and q-axis reactances, $[p u]$ Characteristic reactance (Ref. [1]), [pu] or [ $\Omega]$ Synchr., trans. \& subtrans. d-axis react., [pu] Synchr. and subtrans. q-axis reactances, $[p u]$ Stator phase $a, b$ and $c$ current, $[p u]$ or $[A]$ Damper bar d- \& q-axis current, $[p u]$ or $[A]$ Stator armature d- \& q-axis current, [pu] Rotor field winding current, $[p u]$ or $[A]$ Damper winding d- \& q-axis resistance, $[p u]$ Armature \& field winding resistance, $[p u]$ Laplace operator, $\frac{d}{d t},[\mathrm{rad} / \mathrm{s}]$

Poles of the armature transfer function, $[\mathrm{rad} / \mathrm{s}]$ Stator armature d- \& q-axis voltage, [pu]

\section{INTRODUCTION}

$\mathbf{T}$ $\mathrm{HE}$ analysis of transient behavior of wound-field synchronous machines (WFSMs) requires precise knowledge of the characteristic quantities of the machine [2], [3], [4], [5], [6]. In particular, the estimation of the saturated subtransient reactances $x_{d}^{\prime \prime}$ and $x_{q}^{\prime \prime}$ are essential for the calculation of the electrical and mechanical integrity of the machine and

Frederic Maurer and Jonas Kristiansen Nøland are with the Department of Electric Power Engineering, Norwegian University of Science and Technology (NTNU), Trondheim, 7034, Norway, (e-mail: frederic.maurer@ntnu.no). its shaft line during severe transients such as under threephase or two-phase short-circuits. There are several testing methods available to obtain these quantities through normal procedures [7], where the DC decay method represents a nonconventional alternative [8]. In a DC decay test, the electrical machine has a locked rotor, with the rotor aligned with the direct or quadrature axis (i.e., (d- and q-axis) [9], [10], [11], [12], [13], [14], [15], [16], [17]. Then, DC current is injected in two phases of the machine armature (by closing $S W_{1}$ having $S W_{2}$ open), with a short-circuited field winding (see Fig. 1). After stationary conditions are achieved, the armature winding is then suddenly short-circuited with a circuit breaker (closing $S W_{2}$ ), and the decaying current $\left(i_{f}\right.$ and $i_{a}$ or $i_{b}$ ) is recorded using a current probe. From these measurements, the parameters of the equivalent diagram are identified. This procedure requires less time, less power, and a significantly smaller short-circuit breaker than other methods, e.g., the three-phase short-circuit test. However, the most difficult part of the DC decay methods lies in the rotor alignment process with the d- or q-axis, which is very difficult to perform for high-power facilities. In order to enhance the applicability of these tests, the challenge in aligning the rotor has to be solved, where simple techniques allowing for arbitrary rotor positions are needed.

Earlier works assumed the rotor to be aligned with the dor q-axis, or provide a procedure to align it [9], [10], [11], [12], [13], [14], [15], [16], [17], which is nearby impossible to achieve for high power units. However, recent contributions take another path to simplify the measurements, allowing for arbitrary rotor position [18], [19], [20] but lacks comparison with a sudden short-circuit nor provide any complete study about precision in the case of a practical application. Other contributions analyze other aspects of these approaches. In [9], equivalent diagram parameters are identified using low- 
pass functions. Then, in [10], [14], an FFT is used for the time signals, as also made in the SSFR tests [21]. The characteristic quantities can also be estimated by curve-fitting techniques of the measured decay current, where the poles and zeros of the transfer function are identified, which indirectly reveals the characteristic quantities [10], [15]. Genetic algorithms have also been introduced on this matter [12]. Moreover, in [13], a combination of genetic and Gauss-Newton algorithms is used for parameter estimation. By minimizing an objective function [16], [17], using Levenberg-Marquardt's algorithm, one can also determine the characteristic quantities. Already [22] presented a method to obtain the characteristic quantities for any rotor position by identifying them using a similar approach as utilized in [10], [15]. However, the modeling of the shortcircuit is not precise enough so that some discrepancies appear between the identified parameters and the theoretical ones.

This paper starts by extending the method described in [19] and applies it in a simulation study to analyze the optimal number of measurements and recordings (i.e., angular spacing between the tests and sampling frequency of the measured signals) to give enough practical details so that a real application in a power plant is possible. The objective is to figure out the lowest number of measurements needed to obtain the equivalent diagram with a given precision is presented from these data. By further expanding these aims, the equivalent diagram of a laboratory WFSM is measured and validated by simulation and then compared with the one obtained by a three-phase short-circuit test to give a hook point towards the gold standard and allow to give enough confidence in this method so that it could be widely used. Finally, it draws some conclusions about different equivalent diagram's practicality. Even though considerations on the WFSM's maximum exerted torque has been made, the paper does not consider its dynamic speed response, as a high inertia is assumed. Moreover, the torque-angle characteristics can be indirectly found through the parameter identification.

The paper is organized with the following structure. Section [I] recalls briefly the main mathematical expressions, identification algorithms and measuring methods developed in [19], [23]. Then, Section III presents the results of a parameter identification robustness study simulating the machine SM1 (a high power WFSM machine with known design values, refer to table $\mathrm{I}$ and $\mathrm{II}$ for the definition of the rating and parameter of this machine), which has well-established and measured parameters prior to this paper [19]. In addition, the measurements are extracted from another machine (SM2 the highest power WFSM machine at the laboratory, refer to table $[\mathrm{I}$ and $\mathrm{VII}$ for the definition of the rating and measured parameter ${ }^{1}$ are presented in Section IV. Both machines SM1 and SM2 are specified in Table I Finally, Section V concludes the paper and discusses future research.

\section{Theoretical BACKGRound OF DC DeCAY}

Starting from the voltage equations for $u_{a}$ and $u_{b}$, presented in [24], with $i_{c}=0$ and $i_{b}=-i_{a}$, one can obtain the transfer function for the test circuit presented in Fig. 1. (a). This transfer

\footnotetext{
${ }^{1}$ No design values are available for this machine.
}

(a)

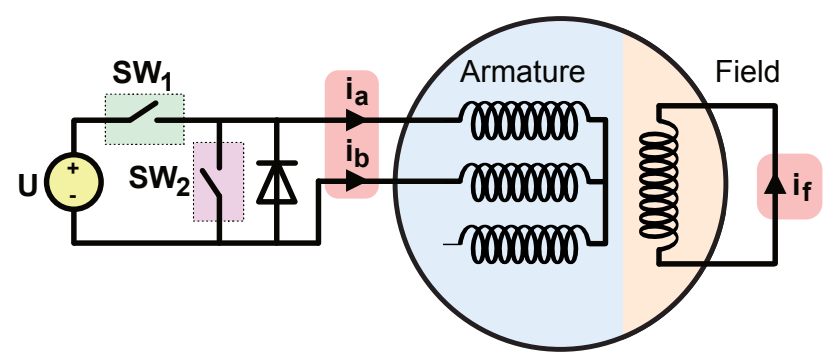

(b)

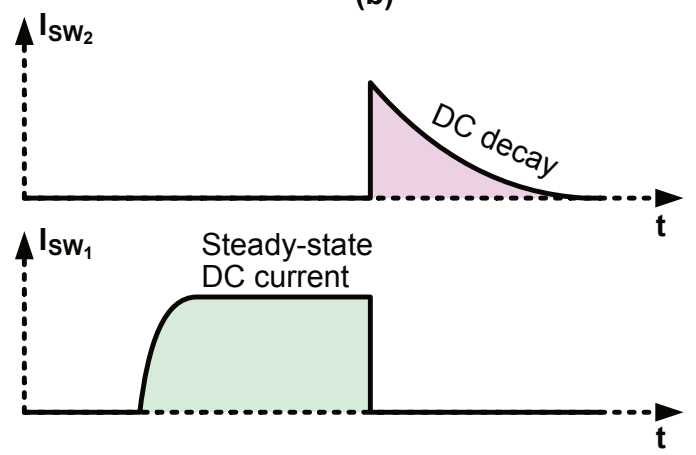

Fig. 1. (a): Experimental test scheme for the DC decay method for SM2. (b): Current through the DC-decay switches, adapted from [19].

TABLE I

SynCHRONOUS MACHINE'S RATED DATA - SM1 \& SM2

\begin{tabular}{|c|l|l|l|}
\hline Symbol & Quantity & SM1 & SM2 \\
\hline$S$ & apparent power & $180.0 \mathrm{MVA}$ & $2.3 \mathrm{kVA}$ \\
$U_{s}$ & stator voltage & $13.8 \mathrm{kV}$ & $380 \mathrm{~V}$ \\
$I_{f 0}$ & no-load field current & $1050.0 \mathrm{~A}$ & $2.0 \mathrm{~A}$ \\
$n$ & mechanical speed & $150 \mathrm{r} / \mathrm{min}$ & $1500 \mathrm{r} / \mathrm{min}$ \\
$f_{s}$ & stator frequency & $50 \mathrm{~Hz}$ & $50 \mathrm{~Hz}$ \\
\hline
\end{tabular}

function considers one damper circuit per axis, corresponding to the modeling of large WFSMs with laminated salient poles with a copper bar damper windings $[25]^{2}$ Herein, $U$ is the voltage of the DC voltage source utilized in the experiment, which drives a measurable stator current $i_{a}$. In order to obtain the reactance $x_{c}$, the field current $\left(i_{f}\right)$ is also recorded (as indicated). $S W_{1}$ and $S W_{2}$ are the switches used for this test.

To perform the test (refer to Fig. 11, first, $S W_{1}$ is closed while $S W_{2}$ remains open, enabling a DC-current to flow in the armature winding. After reaching a steady current, switch $S W_{2}$ is closed and $S W_{1}$ is opened at the same time. This way of handling the switches is shown in Fig. 1 1 (b). The decay of the stator current is then recorded using a transient recorder. Opening $S W_{1}$ is only necessary to protect the DC voltage source from any damages. Finally, when the armature current is zero, $S W_{2}$ can be opened.

The transfer function of the setup described in Fig. 1 assumes small variations, given by [19]

$$
i_{a}(s)=-i_{b}(s)=\frac{Y_{f}(s) Y_{Q}(s)}{Y_{a b}(s)} u_{a b}(s)
$$

\footnotetext{
${ }^{2}$ This type of machine has been handpicked, as it represents more than $95 \%$ of the installed power generation facilities in hydropower.
} 
TABLE II

Reference Standard Parameter Values for Synchronous Machine SM1

\begin{tabular}{|c|c|c|c|c|c|c|c|c|c|c|c|}
\hline$x_{d}$ & $x_{d}^{\prime}$ & $x_{d}^{\prime \prime}$ & $x_{q}$ & $x_{q}^{\prime \prime}$ & $x_{c}$ & $T_{d o}^{\prime}$ & $T_{d o}^{\prime \prime}$ & $T_{d}^{\prime}$ & $T_{d}^{\prime \prime}$ & $T_{q o}^{\prime \prime}$ & $T_{q}^{\prime \prime}$ \\
\hline $1.0250 \mathrm{pu}$ & $0.2960 \mathrm{pu}$ & $0.1980 \mathrm{pu}$ & $0.7090 \mathrm{pu}$ & $0.2020 \mathrm{pu}$ & $0.1150 \mathrm{pu}$ & $8.9095 \mathrm{~s}$ & $0.0417 \mathrm{~s}$ & $2.5630 \mathrm{~s}$ & $0.0280 \mathrm{~s}$ & $0.1088 \mathrm{~s}$ & $0.0310 \mathrm{~s}$ \\
\hline
\end{tabular}

where $Y_{a b}(s)$ is defined as

$$
\begin{aligned}
Y_{a b}(s) & =2\left[r_{s}+\frac{1}{3} s\left(\alpha^{2} \frac{x_{d}}{\omega_{n}}+\beta^{2} \frac{x_{q}}{\omega_{n}}\right)\right] Y_{f}(s) Y_{Q}(s) \\
& -Y_{Q}(p) \frac{2}{3} \frac{\alpha^{2} x_{a d}^{2}}{\omega_{n}^{2}}\left(\frac{1+s T_{\sigma D}}{r_{f}}+\frac{1+s T_{\sigma f}}{r_{D}}\right) s^{2} \\
& -Y_{f}(s) \frac{2}{3} \frac{\beta^{2} x_{a q}^{2}}{r_{Q} \omega_{n}^{2}} s^{2}
\end{aligned}
$$

with $\alpha=\cos (\theta)-\cos \left(\theta-\frac{2 \pi}{3}\right)$ and $\beta=\sin (\theta)-\sin \left(\theta-\frac{2 \pi}{3}\right)$. Moreover, the polynomials $Y_{f}(s)$ and $Y_{Q}(s)$ are expressed as

$$
\begin{aligned}
Y_{f}(s) & =\left(1+s T_{d 0}^{\prime}\right)\left(1+s T_{d 0}^{\prime \prime}\right), \quad \text { and } \\
Y_{Q}(s) & =1+s T_{q 0}^{\prime \prime} .
\end{aligned}
$$

Eq. (1) has identifiable poles found in eq. (2), yielding

$$
Y_{a b}(s) \propto\left(s+s_{1}\right)\left(s+s_{2}\right)\left(s+s_{3}\right)\left(s+s_{4}\right) .
$$

The coupling between the two axes is non-linear and takes place in eq. (1) for $Y_{a b}(s)$ through the constants $\alpha$ and $\beta$. In order to obtain the equivalent circuit for both axes, one must consider the angular variation ("root-locus") of the four zeros of $Y_{a b}(s)\left(s_{1}, s_{2}, s_{3}\right.$, and $\left.s_{4}\right)$, according to eq. 5. This rootlocus of the four zeros exhibits four minima and four maxima, which correspond to a zero or pole of the transfer functions in the d- or q-axis [23]. From those poles and zeros, one can deduce the equivalent diagram. Moreover, there will be some pole/zero simplifications, as the transfer function for a machine merely oriented with one axis has fewer poles and zeros than the transfer function described in eq. (1). This fact complicates the estimation of the maxima and minima of the root-locus and influences the identified characteristic quantities, which is further explored in Section III. The parameter identification algorithm follows strictly the steps described in [19], which includes removing outliers and other specific techniques to enhance the precision of the algorithm.

The determination of $x_{c}$ is done using a similar procedure as presented in [1]. The transfer function between the d-axis armature current and the field current is given by

$$
i_{f}(s)=-\frac{s x_{a d}\left(1+s T_{\sigma D}\right)}{r_{f} \omega_{n}\left(1+T_{d 0}^{\prime}\right)\left(1+T_{d 0}^{\prime \prime}\right)} i_{d}(s) .
$$

The time constant $T_{\sigma D}$ is identified using $i_{f}$. By assuming a certain value of $x_{c}$, the equivalent circuit is computed using the identified characteristic quantities, where $T_{\sigma D}$ is computed from the equivalent circuit. If the error between the identified $T_{\sigma D}$ and the computed one is less than a given threshold, then the assumed value of $x_{c}$ is correct. If the error is too high, then the assumed value of $x_{c}$ is increased by a given step, and the procedure is repeated up to convergence.

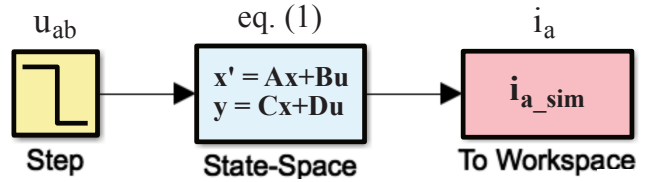

Fig. 2. MATLAB state-space simulation model. Adopted from [19].

\section{Parameter Identification Robustness Study}

In order to gain new insight into the sensitivity of the DC decay method, a robustness study was carried out in the simulation-based environment for machine SM1. The simulation setup in the MATLAB/Simulink environment is shown in Fig 2. The transfer function of eq. (1) was used for the machine SM1, with parameters specified in Table II. Results are obtained assuming ideal switches, switching simultaneously, with no transition time. All the equations are linear, with the voltage source element having a constant voltage. The steady-state current $\left(i_{a}\right)$ is $100 \mathrm{~A}$ and the voltage step in $u_{a b}$ is $0.529 \mathrm{~V}$ (i.e., corresponding to $100 \mathrm{~A}$ ). The transfer function was formulated in a state-space representation that performed the calculation. All the simulations had the same initial conditions. The rotor angle is an input parameter as well as the sampling frequency Both are adjusted to meet the goal of this study. The white-noise is modeled using the ad hoc element present in the MATLAB environment.

The sensitivity of recording was studied in the MATLAB environment, varying the sampling frequency from $1 \mathrm{kHz}$ to $5 \mathrm{kHz}$. Fig. 3 show the influence of the sampling period on the point-by-point capturing of four poles as a function of rotor angle. The exact values of the poles to be identified are detailed in Table III A variation of the sampling frequency between $1 \mathrm{kHz}$ and $5 \mathrm{kHz}$ induces a variation of the radius of convergence of the identification algorithm, which was about $10^{\circ}$ for the $s_{1}$ pole, $5^{\circ}$ for the $s_{2}$ pole, $3^{\circ}$ for the $s_{3}$ pole and $5^{\circ}$ for the $s_{4}$ pole. The sampling frequency of $1 \mathrm{kHz}$ creates new identification errors, e.g., for angles of $150^{\circ}$ and $330^{\circ}$ for the $s_{2}$ pole. This effect is related to the number of samples available to converge the non-linear algorithm so that a toosmall sampling frequency should be avoided.

The errors are the highest for electrical angles in the region around $60^{\circ}, 150^{\circ}, 240^{\circ}$, or $330^{\circ}$. This effect is related to the pole/zero simplification, which prevents the convergence of the curve fit algorithm. These regions, where the error is large, remain visible when the sampling frequency is varied. The error increase is important for the $s_{2}$ pole, which can induce an error when obtaining the maxima and minima of

\footnotetext{
${ }^{3}$ This frequency swipe have been chosen as it represents a "classical" frequency range of transient recorder used in high power machines. In addition, several simulations showed that increasing the sampling frequency doesn't improve the identification quality especially when adding noise.
} 
TABLE III

Obtained Poles of SM1 From Ref. Values Using EQs. 27- (5)

\begin{tabular}{|c|r|r|r|}
\hline Pole & Minima & Maxima & Mean \\
\hline$s_{1}(\theta)$ & $-37.1292 \mathrm{rad} / \mathrm{s}$ & $-35.1303 \mathrm{rad} / \mathrm{s}$ & $-36.3109 \mathrm{rad} / \mathrm{s}$ \\
$s_{2}(\theta)$ & $-23.9775 \mathrm{rad} / \mathrm{s}$ & $-9.1961 \mathrm{rad} / \mathrm{s}$ & $-16.6623 \mathrm{rad} / \mathrm{s}$ \\
$s_{3}(\theta)$ & $-2.8386 \mathrm{rad} / \mathrm{s}$ & $-1.0174 \mathrm{rad} / \mathrm{s}$ & $-1.6916 \mathrm{rad} / \mathrm{s}$ \\
$s_{4}(\theta)$ & $-0.1122 \mathrm{rad} / \mathrm{s}$ & $-0.1012 \mathrm{rad} / \mathrm{s}$ & $-0.1065 \mathrm{rad} / \mathrm{s}$ \\
\hline
\end{tabular}

TABLE IV

Pole Estimation's Root Mean Square Error as Functions of SAMPLing TIME AND FREQUENCY WITH DATA GIVEN IN FIg. 3

\begin{tabular}{|c|r|r|r|r|}
\hline$T_{s} / f_{s}$ & $s_{1}^{*}(\theta)$ & $s_{2}^{*}(\theta)$ & $s_{3}^{*}(\theta)$ & $s_{4}^{*}(\theta)$ \\
\hline $0.2 \mathrm{~ms} / 5.00 \mathrm{kHz}$ & 0.41535 & 2.86104 & 0.00152 & 0.00002 \\
$0.3 \mathrm{~ms} / 3.33 \mathrm{kHz}$ & 3.74464 & 3.72127 & 0.10771 & 0.01188 \\
$0.4 \mathrm{~ms} / 2.50 \mathrm{kHz}$ & 3.74588 & 3.72658 & 0.10771 & 0.01188 \\
$1.0 \mathrm{~ms} / 1.00 \mathrm{kHz}$ & 3.77047 & 4.05882 & 0.10776 & 0.01188 \\
\hline
\end{tabular}

this pole. The error is highest with the lowest sampling rate $(1 \mathrm{kHz})$. However, the value of the error is not identical for all poles. The maximum value of the error is about $10 \%$ for the $s_{1}$ pole, approximately $50 \%$ for the $s_{2}$ pole, while it is less than $1 \%$ for the $s_{2}$ pole, and finally, less than $0.2 \%$ for the $s_{4}$ pole. The higher the sampling frequency, the smaller the error, which can be clearly seen in the root mean square errors (RMSEs) given in Table IV.

Another important characteristic of the DC decay is the influence of the number of measurements, i.e. the number of short circuits to be performed over $360^{\circ}$, on the identification of the maxima / minima of the poles. The angular pitch was varied according to the following list: $1^{\circ}$ (360 test), $2^{\circ}$ (180 tests), $5^{\circ}$ (72 tests), $10^{\circ}$ (36 tests), $15^{\circ}$ (24 tests), and $20^{\circ}(18$ tests), $25^{\circ}$ (14 tests) and $30^{\circ}$ (12 tests).

In addition to the number of tests, the influence of the harmonic content of the fitting function is also needed to obtain the minimum and maximum values of the poles. Table $\mathrm{V}$ shows the 13 cases studied for the variation of the harmonic content. However, the addition of odd harmonics makes no physical sense, other than to possibly help the curve fit algorithm to converge. The poles are approximated as a Fourier series, where $s_{1}$ could be formulated

$$
s_{1}(\theta) \approx \frac{a_{0}}{2}+\sum_{k=1}^{n} a_{n} \cos (n \theta)+\sum_{k=1}^{n} b_{n} \sin (n \theta),
$$

and likewise for the other poles $s_{2}, s_{3}$, and $s_{4}$, respectively.

Fig. 4 shows the effect of varying the harmonic content and the number of measurements on the error identifying the maxima/minima of the poles. In particular, for the angular steps of $25^{\circ}$ and $30^{\circ}$, the method does not reliably identify values from the curves given by the curve fit approach. Interestingly, in most cases, an increase in harmonic content leads to a reduction in the identification error. This error fluctuates but converges towards a certain value $\left(s_{1}\right.$ and $s_{3}$ pole curves). In almost all cases, adding odd harmonics to the curve fit does not improve the result. Consequently, the addition of these harmonics is not necessary.

In the case of the minimum value of the $s_{4}$ pole, increasing
TABLE $\mathrm{V}$

13 Cases of Harmonic Content for Fitting Function in EQ. 7

\begin{tabular}{|l|l|l|}
\hline Case number & Even harmonics & Odd harmonics \\
\hline$\# 1$ & $k=2,4$ & - \\
$\# 2$ & $k=2,4,6$ & - \\
$\# 3$ & $k=2,4,6$ & $k=1$ \\
$\# 4$ & $k=2,4,6$ & - \\
$\# 5$ & $k=2,4,6,8$ & $k=1$ \\
$\# 6$ & $k=2,4,6,8$ & $k=1,3$ \\
$\# 7$ & $k=2,4,6,8$ & - \\
$\# 8$ & $k=2,4,6,8,10$ & $k=1$ \\
$\# 9$ & $k=2,4,6,8,10$ & $k=1,3$ \\
$\# 10$ & $k=2,4,6,8,10$ & - \\
$\# 11$ & $k=2,4,6,8,10,12$ & $k=1$ \\
$\# 12$ & $k=2,4,6,8,10,12$ & $k=1,3$ \\
$\# 13$ & $k=2,4,6,8,10,12$ & $k=1,3$ \\
\hline
\end{tabular}

the harmonic content increases the error. Although the absolute value of the error is almost zero, it should be remembered that the most probable solution is not necessarily the one with the highest case number. It is, therefore, necessary to check which curve fit gives the most probable value. The identification error is maximal for the $s_{3}$ pole, and it is about 100 times less for the $s_{4}$ pole. The identification of the maximum and minimum of the $s_{3}$ pole has a maximum error because the curve showing the variation of the pole as a function of electrical angle does not have a sinusoidal trace. In general, the error is not of the same order of magnitude for all four poles. The maximum error is $0.25 \%$ for the $s_{1}$ pole, $1.4 \%$ for the $s_{2}$ pole, $4.5 \%$ for the $s_{3}$ pole, and $0.05 \%$ for the $s_{4}$ pole.

In addition to the analysis above, two additional effects have been studied. One is the effect of a non-constant angular pitch, and the other is the effect of a parity propagation of the measurement points. These two additional effects only have a practical meaning, reducing and simplifying the measurements. The non-constant angular step does not lead to any notable difference compared to the results in Fig. 4 The errors fall within the limits of the Fig. 4. This effect was studied for an angular step of $15^{\circ}+5^{\circ} 4$ and for a sampling period of $0.2 \mathrm{~ms}$. The second additional effect is the propagation of measurement points. This involves making, for example, a number of measurements between $60^{\circ}$ and $150^{\circ}$ and using the symmetries present in the variation of the poles to deduce all the other values. This technique requires the angle $\theta$ to be known absolutely [19]. However, it has the disadvantage of propagating the errors of identification of the poles. On the other hand, it introduces perfect symmetries, which can also improve the convergence of the curve fit algorithm. A test was performed for an angular step size of $1^{\circ}$ and a sampling period of $0.2 \mathrm{~ms}$. No significant differences could be found. The errors fall within the limits of the Fig. 4

Finally, in order to study the influence of the SNR on the identification of the equivalent diagram, we performed MATLAB simulations adding Gaussian white noise to the

\footnotetext{
${ }^{4}$ The variation of the angular step was carried out using pseudo-random values uniformly distributed between 0 and 5
} 
Sampling time and frequency $\left(f_{S} \& T_{S}\right)$ :

\begin{tabular}{|c|c|c|c|}
\hline $5.0 \mathrm{kHz}-0.2 \mathrm{~ms}:$ & $3.3 \mathrm{kHz}-0.3 \mathrm{~ms}:$ & $2.5 \mathrm{kHz}-0.4 \mathrm{~ms} \vdots$ & $1.0 \mathrm{kHz}-1 \mathrm{~ms}$ \\
\hline
\end{tabular}
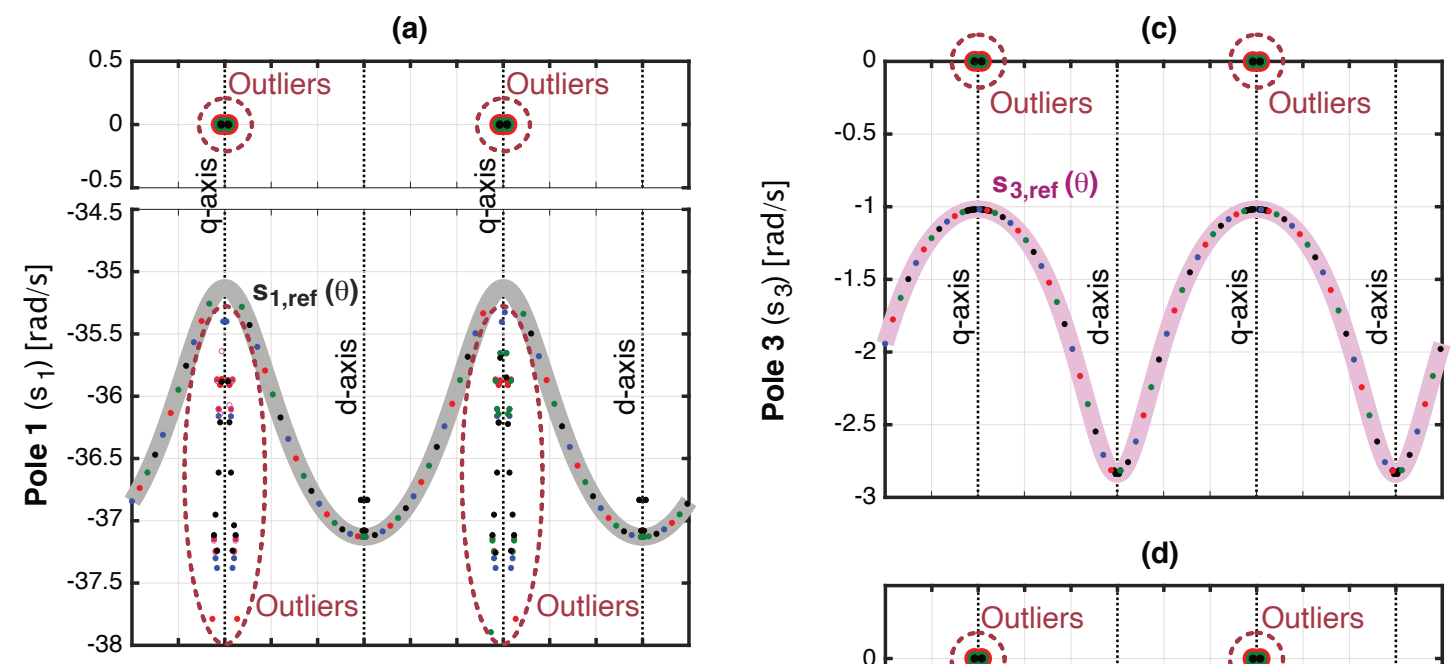

(d)

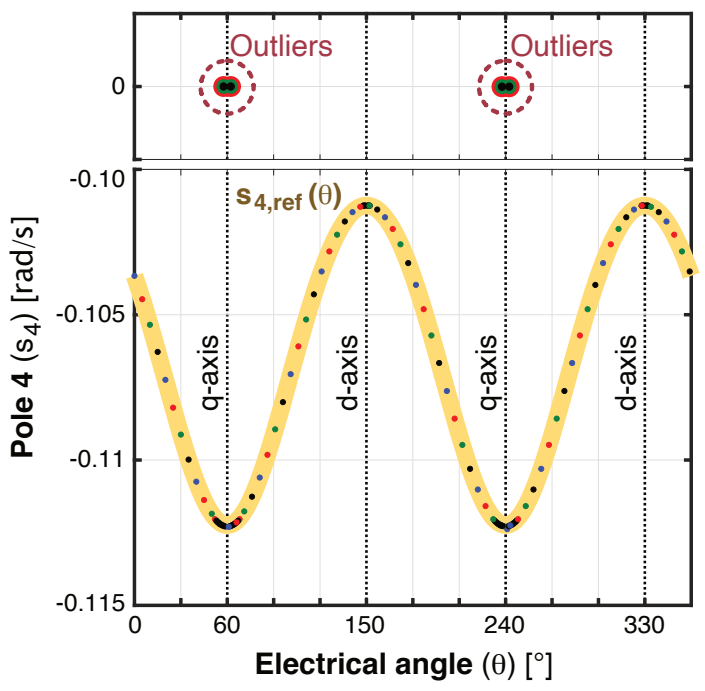

Fig. 3. Influence of the sampling time on the identification of the poles for machine SM1. (a): $s_{1} ;$ (b): $s_{2} ;(\mathbf{c}): s_{3} ;$ (d): $s_{4}$. Error analysis given in Table IV

simulated stator current. We performed 2 sets of simulations, one with a SNR of $40 \mathrm{~dB}$ and another with a SNR of $60 \mathrm{~dB}$. The major impact of the noise lies on the poles $s_{2}$ and $s_{3}$, the other two are quasi-unaffected by the noise. Reducing the angular step below $10^{\circ}$ helps to keep the identification error below $1 \%$, but they can also induce biased-poles near the axis. One should therefore keep distance (approx. $5^{\circ}$ is sufficient) of the axis during the measurements to avoid the biased-poles, that are difficult to remove using the algorithm developed in [19], [23]. Another option would be to increase the robustness of the algorithm in this region, which could be the subject of further research.

Table I in reference [19] presents the results of the identification of characteristic quantities by simulation for the SM1 machine. These results presented show the minimum and maximum errors obtained. The simulations, separately validated using SIMSEN, were performed with a system of state equations written in MATLAB. It can be concluded from the robustness study that the maximum error is less than $1 \%$, which verifies the quality and accuracy of the identification method. It can also be seen that a sampling period of $1 \mathrm{~ms}$ causes an offset on the error. All the errors for a sampling period of $1 \mathrm{~ms}$ are greater than those for a sampling period of $0.2 \mathrm{~ms}$.

Fig. 5 show the effect of a variation in the number of measurement points and a variation in the sampling period on the characteristic standard quantities of the SM1 machine. For each angular step, the maximum and minimum values of the poles chosen are those with the lowest error. Therefore, these are results in the best case. It can be seen that the errors are always less than $1 \%$, highlighting the precision of the method.

In general, the value of the errors does not vary in the same way for all the characteristic quantities. The maximum errors are obtained with high angular steps (few recordings). Therefore, in order to keep the deviation within reasonable limits, no angular step greater than $10^{\circ}$ should be chosen (i.e., less is better, and symmetries can be used to exploit each measurement better). The sampling period does not influence the error uniformly. However, a trend, saying that a low sampling period guarantees a low error, seems to emerge anyway. The non-linear influence of the studied parameters does not allow to draw uni-vocal conclusions. By keeping the signal-to-noise 


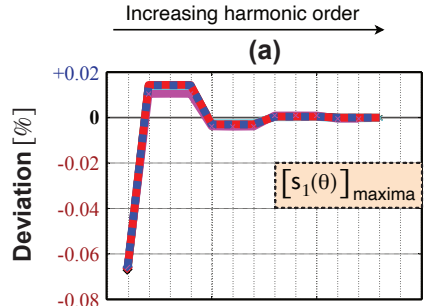

(c)

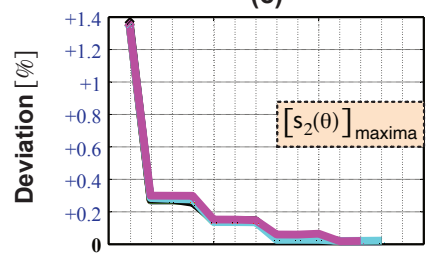

(e)

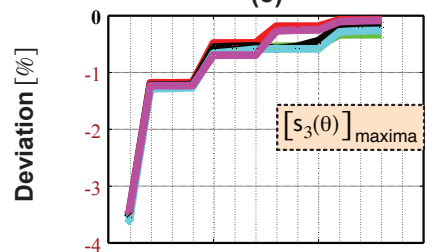

(g)

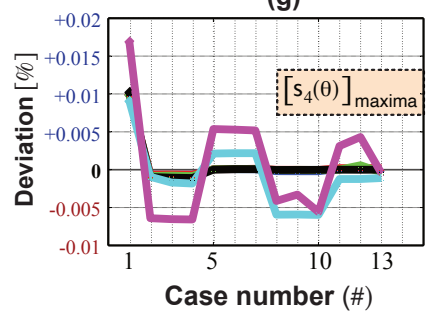

\begin{tabular}{|l|l|l|l|l|l|}
\hline$\Delta \theta=1^{\circ}$ & $\Delta \theta=2^{\circ}$ & $\Delta \theta=5^{\circ}$ & $\Delta \theta=10^{\circ}$ & $\Delta \theta=15^{\circ}$ & $\Delta \theta=20^{\circ}$ \\
\hline
\end{tabular}

Fig. 4. Effect of a variation of the harmonic content on the identification error of the maxima / minima values of the poles for the SM1 machine for a sampling period of $0.2 \mathrm{~ms}$ (reference values given in Table III). Increasing case number means increasing harmonic content, as described in Table V The colors of the curves refers to the the angular step $(\Delta \theta)$, as depicted inside figure. (a): Maxima $s_{1}(\theta)$; (b): Minima $s_{2}(\theta)$; (c): Maxima $s_{1}(\theta)$; (d): Minima $s_{2}(\theta)$; (e): Maxima $s_{3}(\theta)$; (f): Minima $s_{3}(\theta)$; (g): Maxima $s_{4}(\theta)$; (h): Minima $s_{4}(\theta)$.

ratio above $40 \mathrm{~dB}$ guarantees a good identification quality.

\section{EXPERIMENTAL RESULTS}

In this section, explicit validations to the numerical analysis are provided. For this reason, the experimental setup is depicted in Fig. 6 while Fig. 11 shows a schematic layout of the DC decay method, as proposed in [9] and using the measurement methods described in Section I.

As the bearings of the machine are always lubricated, one needs a shaft-blocking device to prevent any rotor movement during the measurements (as indicated in Fig. 6). This device can be very rudimentary, as the torque occurring during these tests is very small. For the DC decay method, the maximal value of the torque $\left(T_{\max }\right)$ achieved during the steady-state condition prior to the decay process is given by

$$
T_{\text {max }}=\frac{4}{9} I_{a}^{2} \alpha \beta\left(x_{d}-x_{q}\right) \cong 0.3 I_{a}^{2}\left(x_{d}-x_{q}\right),
$$

where $I_{a}$ is the stationary pu-value of the stator current. (a)

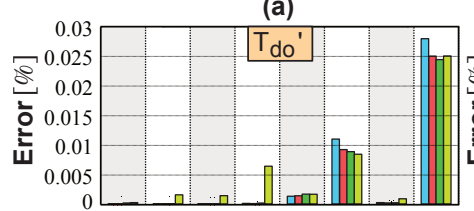

(b)

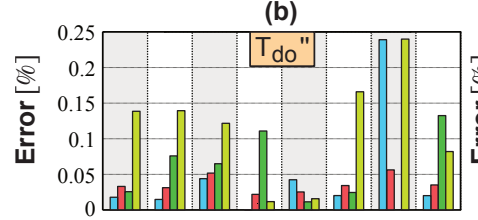

(c)

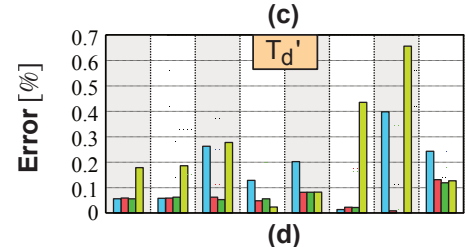

(d)

(e)
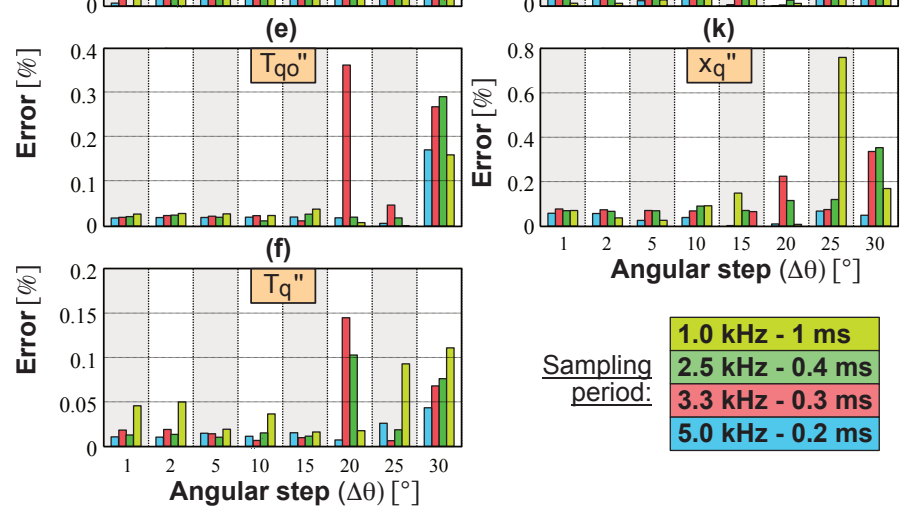

$1.0 \mathrm{kHz}-1 \mathrm{~ms}$

Sampling $2.5 \mathrm{kHz}-0.4 \mathrm{~ms}$

period: $3.3 \mathrm{kHz}-0.3 \mathrm{~ms}$

$5.0 \mathrm{kHz}-0.2 \mathrm{~ms}$

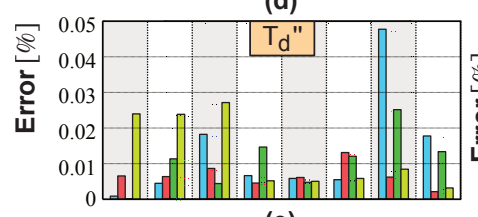

Fig. 5. Effects of a variation in the number of measurement points (i.e., angular step, $\Delta \theta)$ and a variation in the sampling period $\left(T_{e}\right)$ on the characteristic quantities of the direct axis of the SM1 machine. (a): $T_{d o}^{\prime}$; (b): $T_{d o}^{\prime \prime} ;(\mathbf{c}): T_{d}^{\prime} ;(\mathbf{d}): T_{d}^{\prime \prime} ;(\mathbf{e}): T_{q o}^{\prime \prime} ;(\mathbf{f}): T_{q}^{\prime \prime} ;(\mathbf{g}): x_{d} ;(\mathbf{h}): x_{d}^{\prime} ;(\mathbf{i}): x_{d}^{\prime \prime} ;(\mathbf{j}):$ $x_{q} ;(\mathbf{k}): x_{q}^{\prime \prime}$.

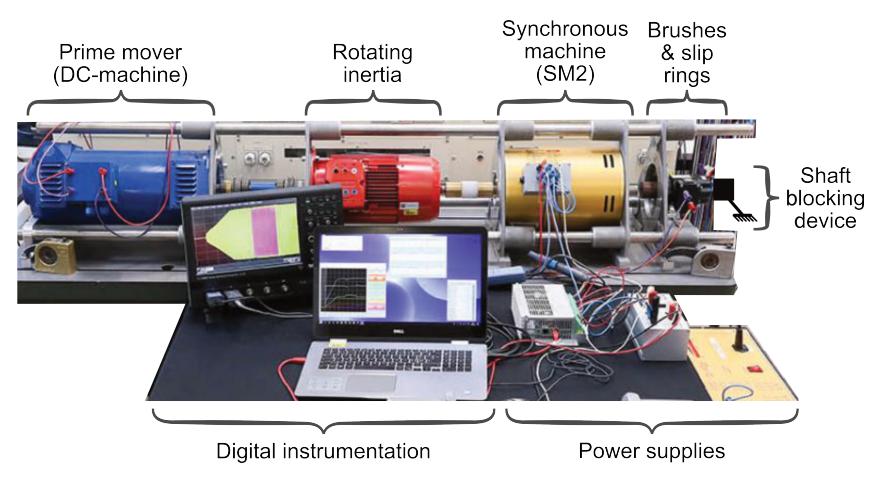

Fig. 6. Experimental setup for the synchronous machine SM2.

SM2 depicted in Fig. 6 is a salient-pole machine with the highest output power available at the laboratory facilities having laminated salient poles. A laminated salient pole (i.e., SM1 and SM2) is composed of stacked and already cut iron 
TABLE VI

ALTERNATIVE METHODS TO OBTAIN THE PARAMETERS OF THE EQUIVALENT DIAGRAM

\begin{tabular}{|c|l|}
\hline Parameters & Method / Norm \\
\hline$x_{d}$ & Permanent short-circuit mode \\
$x_{d}^{\prime}, x_{d}^{\prime \prime}$ & Three-phase short-circuit / application of voltage \\
$T_{d}^{\prime}, T_{d}^{\prime \prime}$ & Three-phase short-circuit \\
$x_{q}$ & Negative excitation \\
$x_{q}^{\prime \prime}$ & Application of voltage \\
$T_{q}^{\prime \prime}$ & Three-phase short-circuit in operation \\
$x_{c}$ & Three-phase short-circuit (excitation current) \\
\hline
\end{tabular}

sheets to form the desired pole shoe shape pressed together with clamping bolts and has a copper damper winding.

In order to sufficiently determine the equivalent circuit for both axes, one usually needs to perform between 10 and 20 measurement processes with a rotor displacement of $180^{\circ}$ between the first and the last recording. As a reference to the parameters obtained via the DC decay method, one can use methods coming from norms (IEEE, IEC). Table VI presents the method and norm used to determine the standard parameters using alternative methods, which have earlier been applied to SM2 for the equivalent diagram's parameters.

\section{A. Recordings of the DC decay Test}

In total, 38 tests at different rotor angles were conducted, covering an angular range of around $180^{\circ}$. Fig. 7 depicts the time evolution of the stator current decay of the SM2 machine during the DC decay test. The shift in response from the $\mathrm{d}$ axis to the q-axis shows peculiar behavior. Instead of having one slip zone, the curves in Fig. 7 show two quite distinct zones, one for positions approaching the d-axis and the other for positions in the region around the q-axis. This effect could be explained by the fact that SM2 is a low-power machine and has a huge saliency effect due to its very narrow air-gap (i.e., $3 \mathrm{~mm}) \sqrt{5}$. The evolution of the sub-transient quantities more or less corresponds to the evolution of the sub-transient quantities of the SM1 machine with a time constant of the d-axis lower than that of the q-axis. Fig. 7 also shows the time evolution of the rotor field current, as the exciter terminals are shortcircuited. For rotor angles between $60^{\circ}$ and $80^{\circ}$, the response has a hump in the sub-transient part of the response, which can not be described using the classical equivalent diagram for WFSMs. This hump can be attributed to the same as the above-mentioned reasons.

Due to the observed large saliency effect, the procedure to determine the equivalent diagram had to be adjusted. After several attempts to use the four-pole transfer function, the approach did not succeed in the entire region. Therefore, near the d-axis, identification was conducted using a three-pole transfer function, while two poles were used near the q-axis. Using these root locus's, one can determine its maximum and minimum as usual from which the equivalent diagram can be computed.

\footnotetext{
${ }^{5}$ This high saliency effect makes that the fundamental theory underpinning the equivalent diagram less precise.
}

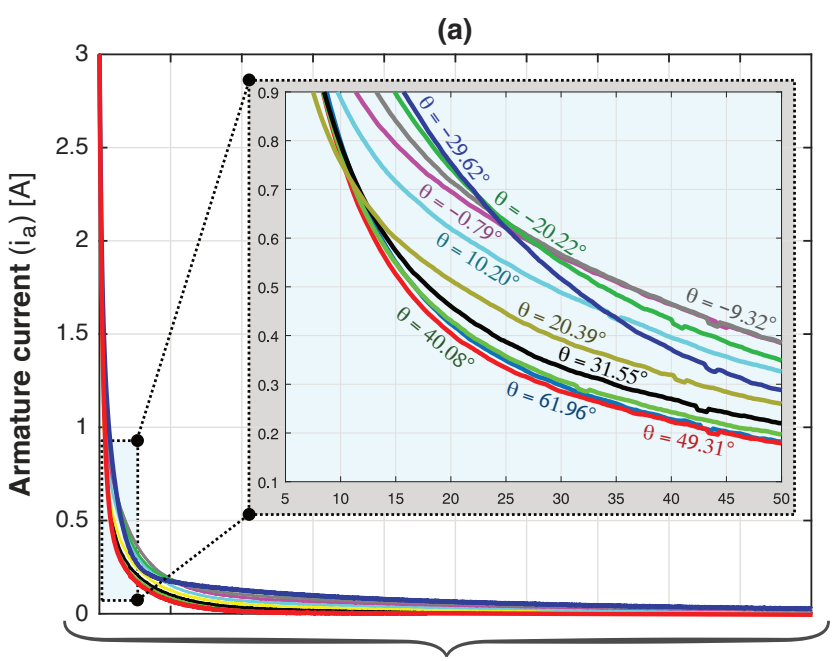

(b)

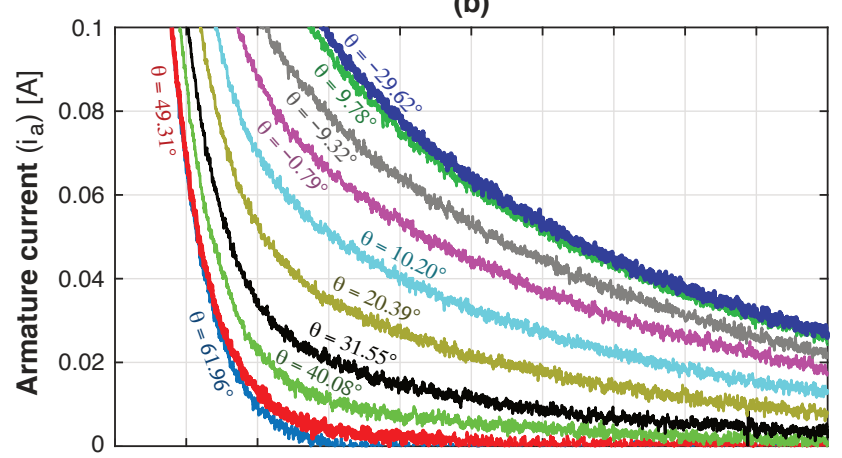

(c)

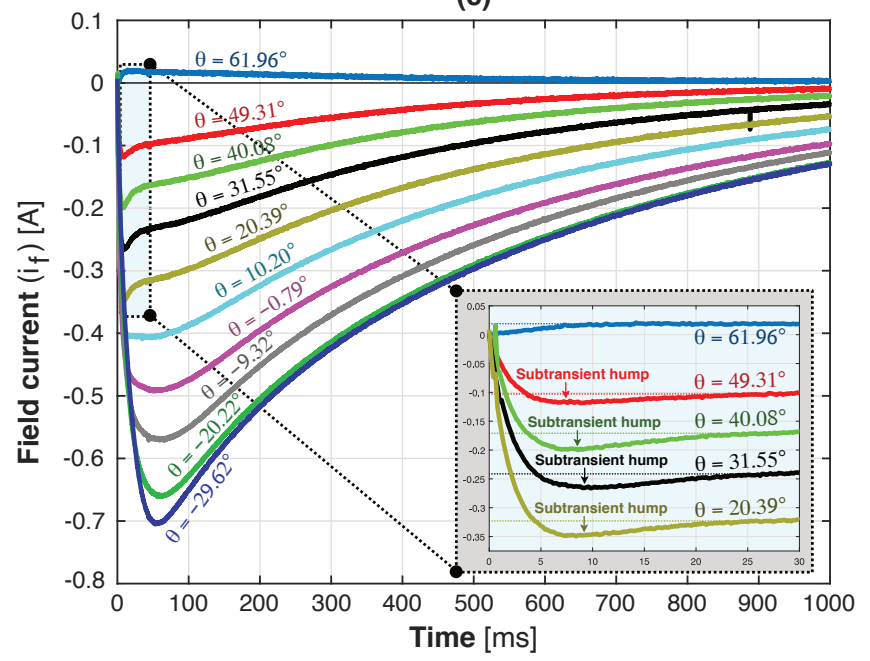

Fig. 7. Measured stator armature current $\left(i_{a}\right)$ and rotor field current $\left(i_{f}\right)$ for different rotor positions in the machine SM2, where stator d- and q-axis are located at $\theta \approx 150^{\circ}$ and $\theta \approx 60^{\circ}$, respectively. The initial armature DC current level was set to $i_{a} \approx-3.0 \mathrm{~A}$ and $i_{b} \approx 3.0 \mathrm{~A}$ at $0 \mathrm{~ms}$, while $i_{c}=0 \mathrm{~A}$ during the whole time interval. The initial field current $\left(i_{f}\right)$ was $0 \mathrm{~A}$ at $0 \mathrm{~ms}$. (a): $i_{a}$ plots in full view. (b): $i_{a}$ plots in zoomed view. (c): $i_{f}$ plots.

\section{B. Assessment of Measurements Against Simulations}

The recorded current decays are used to uncover the limits of simulations using the equivalent diagram in this subsection, and especially considering low-power WFSMs. Using simulations done in the SIMSEN numerical environment, we compare the simulated curves based on the base quantities of SM2 in Table I and its standard parameters in Table VII with 
(a)

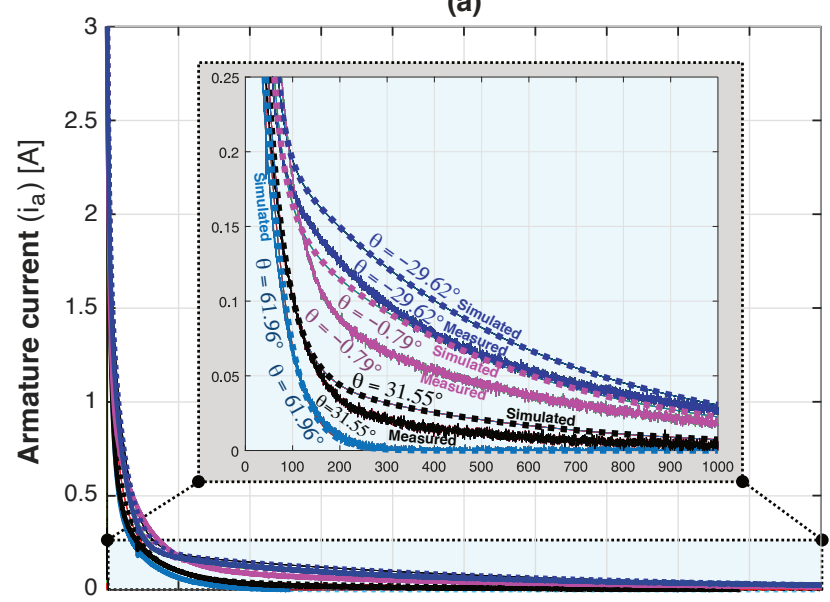

(b)

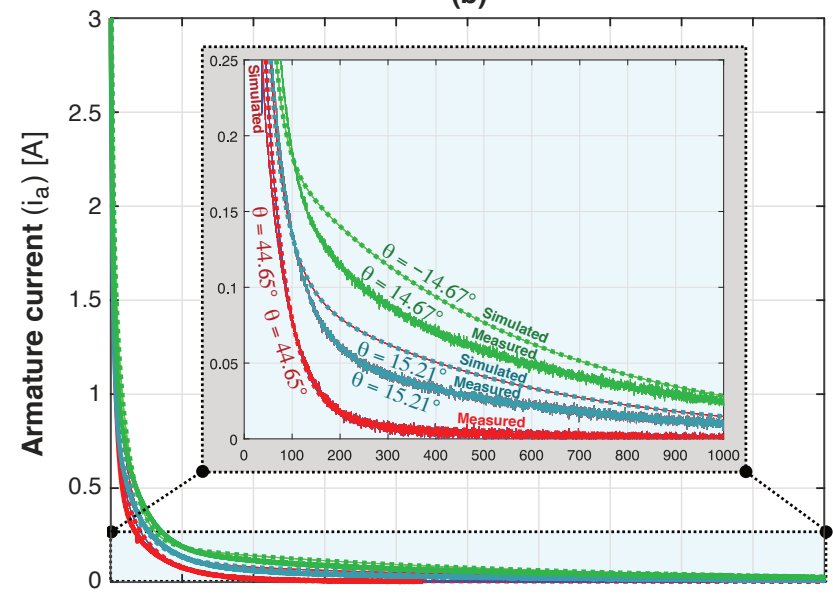

(c)

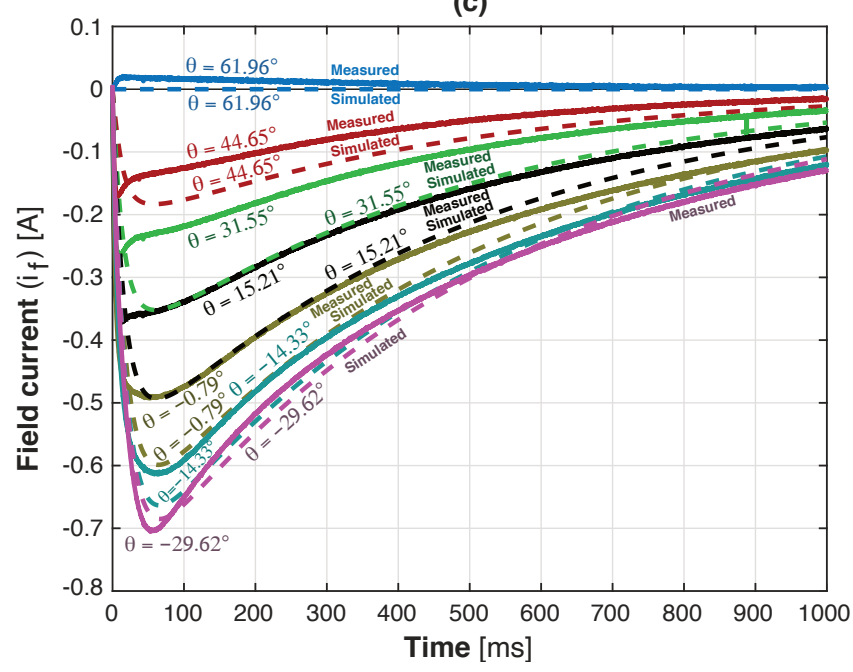

Fig. 8. Comparison between handpicked measured sequences from Fig. 7 against simulated profiles in the SIMSEN numerical environment. (a) $\&$ (b): Stator armature current $\left(i_{a}\right)$. (c): Rotor field current $\left(i_{f}\right)$.

measured currents. When the machine is aligned with the daxis, the difference between simulated and measured armature current is relatively low, revealing the high accuracy of the developed method in this study. The measured curves do not exactly represent the curves in the d-and q-axis; however, the angular deviation is assumed to be small enough to verify the numerical basis for this work.

Fig. 8 show the comparison between simulated and measured currents for angles ranging from $0^{\circ}$ to $90^{\circ}$. The correspondence is excellent for angles in the vicinity of the d-and qaxis. On the other hand, the deviation is more important for angles ranging from $15^{\circ}$ to $45^{\circ}$ because we change the slip zone. These slip zones were not being modeled by the identified machine model. Fig. 8 also shows a comparison between the measured and simulated curves for $i_{f}$. The deviation is greater than that obtained for the stator current. Nevertheless, the agreement between the curves remains satisfactory, especially for the estimation of the maximum value reached by the current. However, it can be seen that the error varies strongly with the electrical angle. Similarly, the estimation of the maximum current value shows errors of the order of $20 \%$. Moreover, the hump observed in the sub-transient part of the response for angles of about $60^{\circ}$ to $80^{\circ}$ is not modeled by the classical equivalent diagram.

Overall, the results are good from a validation point of view. The comparison between the simulation and the measurement showed an excellent agreement between the simulated curves and the measured curves when the machine is shimmed in one of the 2 axes. In between, the agreement is more or less good, as the model used does not perfectly translate the physical reality of this low-power machine. On the other hand, the error in the excitation current is greater but still acceptable for the purpose of machine characterization.

\section{Alternatives for Determination of the Equivalent Circuit}

Table VII compares the obtained equivalent diagram for the DC decay test, and the classical three-phase short-circuit test, respectively. It is very important to note that, as one can see in Figs. 8 and 9 , each method has a very good agreement between measurement and simulation for their respective identified parameters. For the DC decay method, most parameters identified for the d-axis (e.g., $x_{d}, x_{d}^{\prime \prime}, T_{d}^{\prime}$, and $\left.T_{d}^{\prime \prime}\right)$ are perceived as the most accurate, given the nature of the method and the fact that SM2 is a low-power machine with laminated salient poles. However, the value of $x_{d}$ is very high, and $x_{q}$ is very low compared to typical values expected for salient pole machines. These values might be affected due to the signal-to-noise ratio (SNR) at the measurement level is too low in the time interval where these quantities are determined. This condition must be studied with further measurements on a high power machine in a power plant.

For the short-circuit test, the time constants are perceived to be very low compared to "classical values". Given the measurements, it is difficult to compute them with precision, i.e., the results should be taken with a certain precaution. For example, considering the very low value of the sub-transient time constant $\left(T_{d}^{\prime}\right)$, the use of the standardized classical method should be questioned on this basis. In fact, the conventional approaches lead to only a few data points, causing their identified values to be inversely proportional to the voltage, which is the main drawback of the three-phase SC test for low-power machines.

Fig. 9 compares the two sets of parameters in Table VII to predict a sudden short-circuit (SC) at rated voltage, where the 
TABLE VII

IDENTIFIED STANDARd PARAMETERS OF SM2 USING THE DC DECAY TESTS OBTAINED FROM FIG. 6 AND THE SHORT-CIRCUIT TEST IN FIG. 8

\begin{tabular}{|l|c|c|c|c|c|c|c|c|}
\hline Test method & $x_{d}$ & $x_{d}^{\prime}$ & $x_{d}^{\prime \prime}$ & $x_{q}$ & $x_{q}^{\prime \prime}$ & $T_{d}^{\prime}$ & $T_{d}^{\prime \prime}$ & $T_{q}^{\prime \prime}$ \\
\hline DC decay test & $0.7842 \mathrm{pu}$ & $0.2541 \mathrm{pu}$ & $0.1089 \mathrm{pu}$ & $0.2325 \mathrm{pu}$ & $0.1069 \mathrm{pu}$ & $147.2 \mathrm{~ms}$ & $4.7 \mathrm{~ms}$ & $17.7 \mathrm{~ms}$ \\
Three-phase SC test (from [26], 1 pu) & $1.0810 \mathrm{pu}$ & $0.1665 \mathrm{pu}$ & $0.0800 \mathrm{pu}$ & $0.2600 \mathrm{pu}$ & $0.0800 \mathrm{pu}$ & $29.5 \mathrm{~ms}$ & $9.6 \mathrm{~ms}$ & $7.7 \mathrm{~ms}$ \\
Three-phase SC test (from [26], $0.2 \mathrm{pu})$ & $1.2756 \mathrm{pu}$ & $0.2089 \mathrm{pu}$ & $0.1205 \mathrm{pu}$ & $\mathrm{n} / \mathrm{a}$ & $0.1205 \mathrm{pu}$ & $51.8 \mathrm{~ms}$ & $10.7 \mathrm{~ms}$ & $\mathrm{n} / \mathrm{a}$ \\
\hline
\end{tabular}

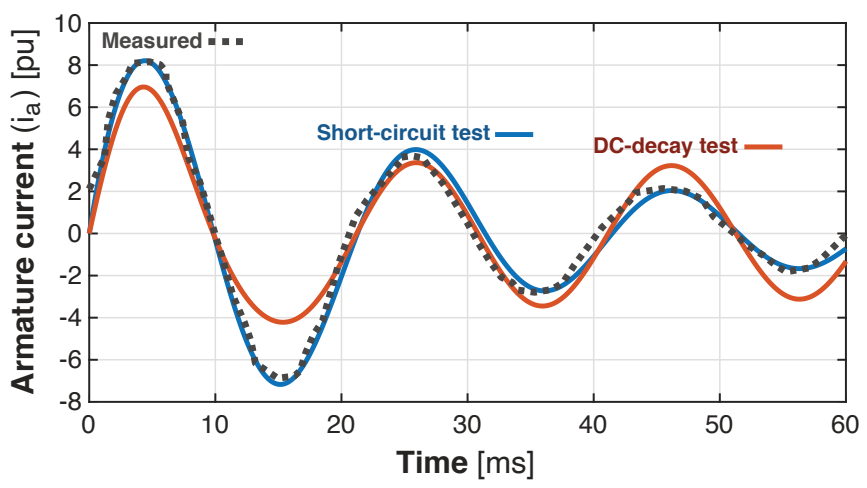

Fig. 9. Armature current response of a sudden three-phase short-circuit at rated voltage $(380 \mathrm{~V})$ and frequency $(50 \mathrm{~Hz})$, with $2.02 \mathrm{~A}$ field current and $1500 \mathrm{r} / \mathrm{min}$ mechanical speed, comparison measurement against simulations using the parameters from the short-circuit test and the DC decay test (given in Table VII , respectively. Measurements taken from the technical report of Wymann [26] for the same machine SM2. The base value of the armature current is $3.5 \mathrm{~A}$.

three-phase SC test has the best precision for this particular case. The difference between the current peak value is around $20 \%$, which is a high value, but still within accepted tolerances according to IEC/IEEE. It is worth noting that the DC decay value cannot accurately model the short-circuit test at a rated voltage (i.e., in saturated mode initially). The most likely explanation for this discrepancy (removing the false value of $x_{d}$ due to the very low SNR) is that the DC decay test cannot saturate the machine as the short-circuit test. Nonetheless, the parameters obtained are outside of the typical range, as expected for smaller machines [27], [28]. This is due to a higher saliency effect that gets influential in low-power machines due to their relatively small air gap. This makes the fundamental theory underlying the equivalent diagram less accurate than for high-power machines with larger air gaps. So the error value of $20 \%$ should be interpreted in this context. Further measurements will be performed on high power machine to confirm the error or not.

Finally, the obtained parameters are inline with those obtained in non-saturated conditions (0.2p.u. of rated voltage Table VII enforcing the fact that the DC-Decay is more than a valable measurement method to obtain the equivalent diagram of a synchronous machine. In addition, the maximal torque amplitude is in the range of 0.2 p.u., while it reaches 8 p.u. (16x the value of the DC-Decay test !) for the sudden SC at rated voltage. This is a game winning advantage as the DC-Decay tests performs at least as well as the sudden SC but represents no risk / no lifetime reduction for the electrical machine and can be therefore conducted without any restriction.

\section{CONClusion}

This paper showcased the main challenges when performing the DC decay test on WFSMs, i.e., the sensitivity to sampling frequency and angular resolution. Furthermore, we could also demonstrate their impact on the identification of the equivalent diagram as well as the sensitivity to measurement noise. As already pinpointed in [19], the DC decay method is not well suited to obtain $x_{d}$ et $x_{q}$ due to the low SNR in the time interval where those values are obtained, as further elaborated in this paper.

We ensured the robustness of the identification algorithm that was utilized, which was also validated under non-ideal conditions. Our identification of the equivalent diagram of a low-power WFSM demonstrated a good agreement between simulation and measurement. However, we also take into account that, as widely known and accepted, the classical equivalent diagram has limitations for smaller machines. In order to get an identification precision below $1 \%$, we recommend getting an SNR above $40 \mathrm{~dB}$, a sampling frequency above $5 \mathrm{kHz}$ and an angular resolution below $10^{\circ}$ with measurement propagation. Due to symmetry considerations, 9 recordings should be minimally sufficient, while 10 to 12 tests will be even better from an accuracy point of view.

Finally, a first attempt to compare a short-circuit test and a DC decay test have been performed comparing data measured at rated voltage, to our best knowledge. We could demonstrate that the DC-Decay test lies in the accepted $20 \%$ current error as per IEC/IEEE even in the saturated case and for a small power machine, where it is known that the parameters do not behave "normally". Comparing the values with the nonsaturated ones also leads to an acceptable discrepancy. The maximal torque amplitude is reduced by factor 16 compared to the sudden gold-standard SC test, which basically cancels any damages on the electrical machine (rotor and winding overhang). This enables to perform the DC-decay without any consequence and leads to results that are of an acceptable quality to determine the equivalent diagram of synchronous machines.

Further measurements on a high-power synchronous machine will be performed to enhance the comparative range of both methods and try to sharpen the equivalence range of both measurement methods.

\section{ACKNOWLEDGMENT}

The authors would like to thank engineer Tino Wymann, Department of Electric Engineering, EPFL, for his valuable contributions to the laboratory experiments conducted as part of the work presented (especially the measurements of the equivalent diagram via other methods). His technical skills in 
instrumentation, creativity in finding practical solutions, flexibility, and interpersonal skills have been of great importance in this context and are highly appreciated.

\section{REFERENCES}

[1] I. Canay, "Determination of model parameters of synchronous machines," in IEE Proc. B (Electr. Power Appl.), vol. 130, no. 2. IET, 1983, pp. 86-94.

[2] B. Zaker, G. B. Gharehpetian, and M. Karrari, "Improving synchronous generator parameters estimation using $d-q$ axes tests and considering saturation effect," IEEE Trans. Ind. Informat., vol. 14, no. 5, pp. 1898 1908,2018

[3] E. d. C. Bortoni, B. T. de Araujo, and J. A. Jardini, "Estimation of quadrature axis synchronous reactance using the constant excitation test," IEEE Power and Energy Tech. Syst. J., vol. 3, no. 2, pp. 43-50, 2016.

[4] M. Micev, M. Ćalasan, D. S. Petrović, Z. M. Ali, N. V. Quynh, and S. H. E. Abdel Aleem, "Field current waveform-based method for estimation of synchronous generator parameters using adaptive black widow optimization algorithm," IEEE Access, vol. 8, pp. 207537 $207550,2020$.

[5] A. Mitra, A. Mohapatra, S. Chakrabarti, and S. Sarkar, "Online measurement based joint parameter estimation of synchronous generator and exciter," IEEE Trans. Energy Convers., vol. 36, no. 2, pp. 820-830, 2021.

[6] E. F. Alves, J. K. Nøland, G. Marafioti, and G. Mathisen, "Online parameter identification of synchronous machines using kalman filter and recursive least squares," in Proc. IEEE 45th Ann. Conf. of Ind. Electron. Soc. (IECON), vol. 1, 2019, pp. 7121-7128.

[7] IEEE, "Ieee guide for test procedures for synchronous machines including acceptance and performance testing and parameter determination for dynamic analysis," IEEE Std 115-2019 (Revision of IEEE Std 115-2009), pp. 1-246, 2020.

[8] I. E. Commission et al., "Rotating electrical machines. part 4: Methods for determining synchronous machine quantities from tests," Norma técnica. Código: IEC, pp. $60034-4,2008$.

[9] E. Boje, J. Balda, R. Harley, and R. Beck, "Time-domain identification of synchronous machine parameters from simple standstill tests," IEEE Trans. Energy Convers., vol. 5, no. 1, pp. 164-175, 1990.

[10] M. Arjona, M. Cisneros-Gonzalez, and C. Hernandez, "Development of a synchronous-generator experimental bench for standstill time-domain tests," J. Appl. Research Tech., vol. 9, no. 2, pp. 117-128, 2011.

[11] F. Sellschopp and M. Arjona, "Dc decay test for estimating d-axis synchronous machine parameters: a two-transfer-function approach," IEE Proc. - Electr. Power Appl., vol. 153, no. 1, pp. 123-128, 2006.

[12] M. Vahedi, A. Hassannia, and H. Lotfian, "Unique solution for dynamic parameters identification of a synchronous machine using dc decay test," J. Electr. Eng., vol. 13, no. 3, pp. 8-8, 2013.

[13] M. Cisneros-Gonzalez, C. Hernandez, R. Escarela-Perez, and M. Arjona, "Determination of equivalent-circuit parameters of a synchronous generator based on the standstill dc decay test and a hybrid optimization method," Electr. Power Comp. Syst., vol. 39, no. 7, pp. 645-659, 2011.

[14] T. Kano, Y. Watanabe, T. Ara, and T. Matsumura, "Calculation of equivalent circuit constants of synchronous machines considering field transient characteristics using dc decay testing method with open and shorted field windings," Electr. Eng. Japan, vol. 178, no. 2, pp. 39-46, 2012.

[15] P. Turner, A. Reece, and D. Macdonald, "The dc decay test for determining synchronous machine parameters: measurement and simulation," IEEE Trans. Energy Convers., vol. 4, no. 4, pp. 616-623, 1989.

[16] L. Majka and D. Szuster, "Application of the stationary de decay test to industrial turbogenerator model parameter estimation," Przeglkad Elektrotechniczny, vol. 90, no. 4, pp. 242-245, 2014.

[17] M. Hasni, O. Touhami, R. Ibtiouen, M. Fadel, and S. Caux, "Synchronous machine parameter identification by various excitation signals," Electr. Eng., vol. 90, no. 3, pp. 219-228, 2008.

[18] F. Maurer, M. T. Xuan, and J.-J. Simond, "Two novel methods for parameter identification of synchronous machine using dc-decay test with rotor in arbitrary position," in Proc. XXII Int. Conf. Electr. Mach. (ICEM), 2016, pp. 633-639.

[19] _ "Two full parameter identification methods for synchronous machine applying dc-decay tests for a rotor in arbitrary position," IEEE Trans. Ind. Appl., vol. 53, no. 4, pp. 3505-3518, 2017.

[20] Y. Ma, L. Zhou, and J. Wang, "Standstill time-domain response parameter estimation of the large synchronous condenser in arbitrary rotor position," IEEE Access, vol. 8, pp. 166047-166059, 2020.
[21] A. Belqorchi, U. Karaagac, J. Mahseredjian, and I. Kamwa, "Standstill Frequency Response Test and Validation of a Large Hydrogenerator," IEEE Trans. Power Syst., vol. 34, no. 3, pp. 2261-2269, May 2019.

[22] L. Vicol, M. Tu Xuan, R. Wetter, J.-J. Simond, and I. Viorel, "On the identification of the synchronous machine parameters using standstill dc decay test." EPFL Lausanne, Tech. Rep., 2006.

[23] F. M. Maurer, "Determination of the parameters of a synchronous machine using dc-decay tests," Ph.D. dissertation, EPFL Lausanne, 2009.

[24] P. C. O. Silva, "Modal analysis applied to the stability study of hydroelectric systems with modular structures," EPFL, Tech. Rep., 2015.

[25] I. M. Canay, "Causes of discrepancies on calculation of rotor quantities and exact equivalent diagrams of the synchronous machine," IEEE Trans. Power App. Syst., vol. PAS-88, no. 7, pp. 1114-1120, 1969.

[26] T. Wymann, P. Allenbach, S. Robert, and J.-J. Simond, "Couplage simsen d'un régulateur de tension." EPFL Lausanne, Tech. Rep., 2008.

[27] J. Martin, C. Tindall, and D. Morrow, "Synchronous machine parameter determination using the sudden short-circuit axis currents," IEEE Trans. Energy Convers., vol. 14, no. 3, pp. 454-459, 1999.

[28] C. Tindall, J. Martin, D. Morrow, and P. Calvert, "Transient characteristics of small salient-pole alternators," IEEE Trans. Energy Convers., vol. 11 , no. 3 , pp. 539-546, 1996.

Frederic Maurer received his M.Sc. degree in 2009 and the Ph.D. degree in 2019 from the Swiss Federal Institute of Technology in Lausanne (EPFL). Since April 2009, he is working for Alstom Hydro (now GE Hydro) in differents positions: R\&D engineer in the generator technology center, Lead electrical engineer, and technical project manager. From February 2021, he is a guest researcher at the Department of Electric Power Engineering, Norwegian University of Science and Technology. His current research interests include circulating current, electromagnetics, improved utilization of electrical machines, and electricity market design. Dr. Maurer regularly serves as a Reviewer for IEEE journals and conferences.

Jonas Kristiansen Nøland (S'14-M'17) was born in Drammen, Norway, in 1988. He received the M.Sc. degree in electric power engineering from the Chalmers University of Technology, Gothenburg, Sweden, in 2013, and the Ph.D. degree in engineering physics from Uppsala University, Uppsala, Sweden, in 2017. Since 2018, he has been an Associate Professor with the Department of Electric Power Engineering, Norwegian University of Science and Technology. His current research interests include excitation systems, hydrogenerators, large AC machines, and enhancing their utilization. Dr. Nøland serves as an Editor for the IEEE TRANSACTIONS ON ENERGY CONVERSION and as an Associate Editor for the IEEE TRANSACTIONS ON INDUSTRIAL ELECTRONICS. 